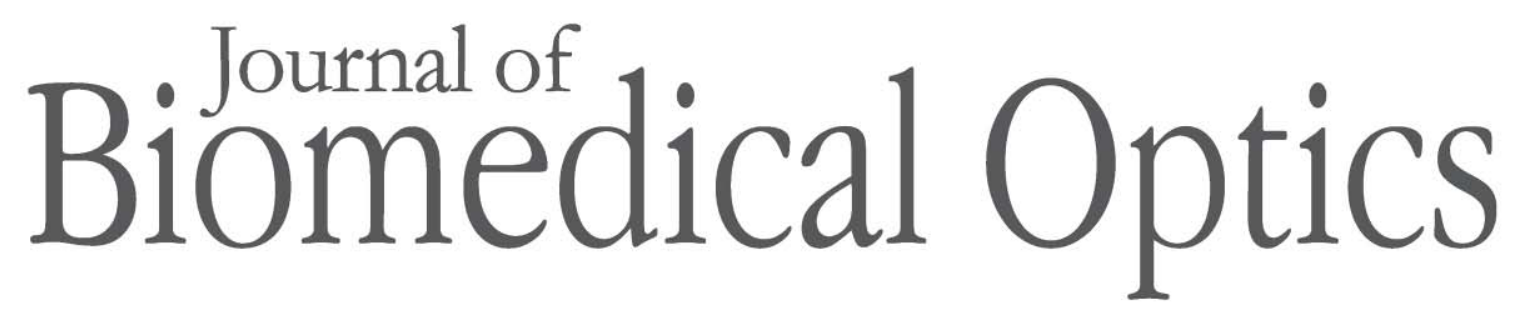

SPIEDigitalLibrary.org/jbo

\title{
Measuring and predicting eyelid spectral transmittance
}

Andrew Bierman

Mariana G. Figueiro

Mark S. Rea 


\title{
Measuring and predicting eyelid spectral transmittance
}

\author{
Andrew Bierman, Mariana G. Figueiro, and Mark S. Rea \\ Rensselaer Polytechnic Institute, Lighting Research Center, 21 Union Street, Troy, New York 12180
}

\begin{abstract}
The purpose of the present study was to objectively quantify the spectral transmittance of the eyelid. Reported here are data acquired using a technique that was developed to provide practical and accurate measurements of eyelid transmittance across the visible portion of the electromagnetic spectrum. The empirical data were analyzed in terms of the absorption and scattering characteristics of the constituents of skin to develop a method for predicting eyelid transmission. Results showed that the eyelid has a much higher optical density at short wavelengths than previously published. The mean \pm standard deviation (s.d.) optical density of the eyelid from 450 to $650 \mathrm{~nm}$ was $2.1 \pm 0.3$ with an optical density range among subjects of approximately 1.0 . The study results indicate that skin pigmentation is poorly correlated with eyelid transmission; eyelid transmission is most affected by wavelength-independent macromolecules in the eyelid as well as its overall thickness. ( 2011 Society of Photo-Optical Instrumentation Engineers (SPIE). [DOI: 10.1117/1.3593151]
\end{abstract}

Keywords: spectral transmittance; wavelength; eyelid; melanin; light.

Paper 11100RR received Mar. 4, 2011; revised manuscript received Apr. 26, 2011; accepted for publication May 3, 2011; published online Jun. 8, 2011.

\section{Introduction}

The human eyelid is not opaque. Every sighted person experiences light sensation through closed eyelids on a sunny day. Following a night of dark adaptation, even dim morning light filtering through curtained windows in a bedroom may be seen through closed eyelids. Retinal light exposure also affects nonvisual systems like alertness, hormone production, and the timing of the sleep-wake cycle. ${ }^{1}$ Recognizing that the eyelid will alter the amount and spectral composition of light incident on the retina, there is every reason to assume that the eyelid-filtered light incident on the retina can stimulate these nonvisual effects. For example, light at night filtered through the eyelid may be used to treat certain circadian sleep disorders, such as delayed sleep. ${ }^{2}$ Thus, it is important to quantify the spectral transmittance of the eyelid to characterize photic stimuli for both the visual and the nonvisual systems.

Toward this end, two techniques have been used to quantify the spectral transmission of the eyelid which include objective $\mathrm{e}^{3,4}$ and psychophysical ${ }^{5}$ methods. The psychophysical method has the advantage of having a high degree of face validity for assessing the effects of eyelid closure on light perception. Drawbacks of this technique are that, to acquire valid data, the experiments are very time consuming and, in the end, have relatively low precision in terms of both spectral resolution and absolute transmittance. Objective measures can be conducted relatively quickly and offer a much higher degree of precision, but extreme care must be taken to ensure that the measurements accurately represent the transmittance of light through the living eyelid without instrumentation artifacts. Also, it should be noted that models of skin reflectance have been developed, ${ }^{6-8}$ but these reflectance models have not been used to model eyelid transmittance, because both spectral reflectance and spectral transmittance are not necessarily correlated and because the eyelid is a unique

Address all correspondence to: Mark Rea, Rensselaer, Polytechnic Institute, Lightning Research Center, 21 Union Street, Troy, New York 12180. Tel: (518) 6877100; Fax: (518) 687-7120; E-mail: ream@rpi.edu. structure, only partially dependent upon the optical properties of the skin.

The present study employed an objective method to measure the spectral transmission of the eyelid. Earlier studies used specially constructed contact lenses coupled to fiber-optic cables to either collect transmitted light ${ }^{4}$ or to provide a source of light behind the eyelid for detection in front of the eyelid. ${ }^{3}$ Typically, optical systems of this basic design have difficulty achieving high optical throughput and consequently have low signal-to-noise ratios. Random noise, such as thermal, amplifier, and photon noise, as well as stray light within the spectrometer, limit accuracy. ${ }^{9}$ Random noise produces uncertainty in the transmittance exhibited as large fluctuations above and below the expected trend in the spectral transmittance curve, while stray light can result in artificially higher spectral transmittance values that approach one as the ratio of stray light increasingly dominates the signal. ${ }^{9}$ For the present study, a different approach to measure the spectral transmission of the eyelid over the visible region of the spectrum was taken, with particular attention to decreasing stray light and increasing the signal-to-noise ratio in the eyelid spectral transmission measurements. A rigid sourcedetector apparatus was designed and built to avoid the losses associated with fiber-optic coupling. A very small, solid-state light source could be located directly behind the eyelid while the detector was located close to the front of the eyelid; this apparatus design provided high optical throughput from 450 to $650 \mathrm{~nm}$. Thus, the acquired data were expected to provide sufficient spectral resolution across this wavelength range for an accurate assessment of individual eyelid spectral transmittance. It was recognized that light transmitted from the back to the front of the eyelid may introduce some artifacts with respect to light from the environment stimulating the retina after passing through the eyelid, if there are differential scattering properties of the various layers comprising the eyelid. ${ }^{10,11}$ This anisotropic effect was assumed to be quite small with respect to the overall spectral transmission through the closed eyelid. In

1083-3668/2011/16(6)/067011/8/\$25.00 (C) 2011 SPIE 
addition to the empirical measurements, it was expected that the acquired spectral transmittance values from individual eyelids could be used as the foundation for a regression model based on the spectral absorbance properties of the known constituent elements of skin, together with inferred scatter characteristics by macromolecules in the eyelid.

\section{Methods}

The measurement apparatus consisted of a spectrometer [Oriel Corporation, Inc., model Multispec 77400 with Instaspec IV linear charge-coupled device (CCD) array detector] with a fiber-optic input and a phosphor-converted, white, light-emitting diode (LED) as the light source. The input end of the spectrometer fiber-optic and the white LED were mounted to a handheld wand that fixed the relative positions of each component. The experimenter could then place the subject's eyelid between the source and the detector components, ensuring a fixed geometry. Figure 1 shows a photograph of the wand device. The wand is tethered to the spectrometer via a 1-m fiber optic cable and to a 3-V, 20-mA power supply via wires that powers the LED.

The LED is a surface mount "chip" type (ROHM Semiconductor model SMLP12W BC7W) measuring $1.0 \mathrm{~mm} \times 0.6 \mathrm{~mm}$ $\times 0.2 \mathrm{~mm}$ (length $\times$ width $\times$ height), and is mounted to a plastic strip 6-mm wide and approximately $0.5-\mathrm{mm}$ thick that extends $22 \mathrm{~mm}$ beyond the end of the wand. Two 30 gauge wires electrically connect the LED to larger wires in the wand. The LED and connecting wires are encapsulated in a dome of clear epoxy covering one side of the plastic strip. The total thickness of the plastic strip and epoxy dome covering the LED and wires (the component placed under the eyelid) was less than $2 \mathrm{~mm}$. During the test, the LED was powered intermittently for periods of less than $20 \mathrm{~s}$ with a maximum power dissipation of $35 \mathrm{~mW}$. This produced a negligible temperature rise during the measurement. Figure 2 shows how the wand device was used to position the LED strip under the eyelid during a measurement.

For this white LED, the spectral output power is essentially zero outside the wavelength range between 450 and $650 \mathrm{~nm}$. An advantage of the limited spectral output of the LED source is that errors due to stray light and incomplete dispersion of the white light by the single grating spectrometer used with the wand

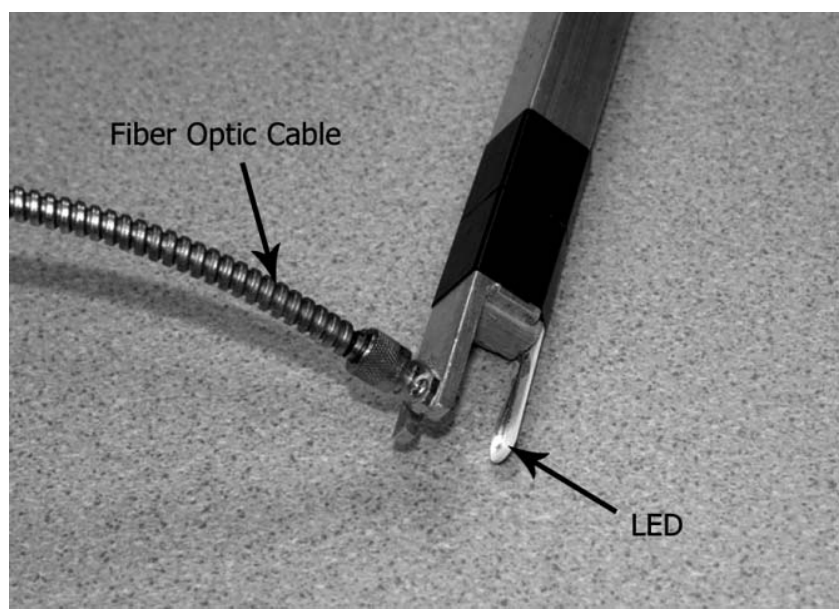

Fig. 1 Wand device for measuring eyelid spectral transmittance.

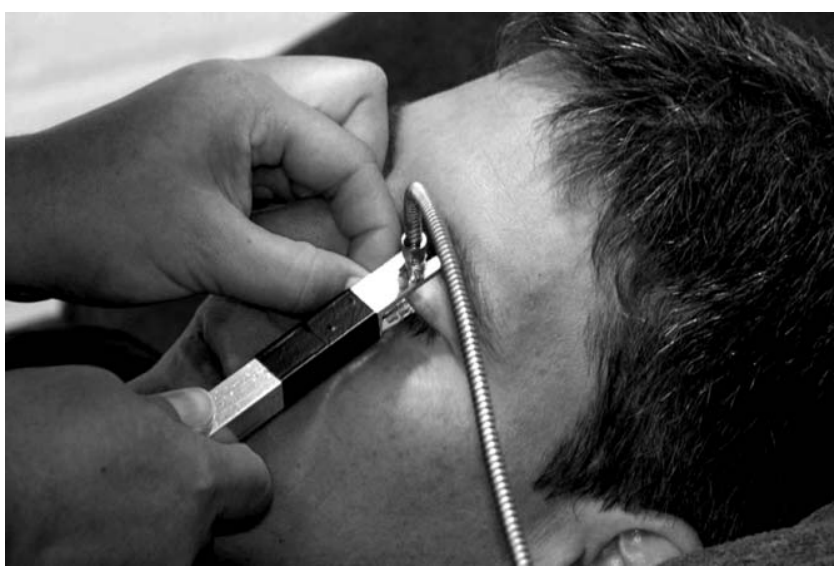

Fig. 2 The wand device being used to measure eyelid spectral transmittance. The LED is positioned under the eyelid and the detector above the eyelid.

device are greatly reduced. In other words, the spectral purity at each measured wavelength interval is much better than if a broader-band emission source, such as an incandescent lamp, had been used.

\section{Diffuse Transmittance Measurement Correction Factor}

The transmittance of a diffuser is measured in terms of the amount of light passing through the medium in the direction of a detector. The transmittance of an ideal diffuser is 0.5 because half the light is scattered away from the detector. The desired quantity of measurement for eyelid transmittance would be its total diffuse transmittance, equal to the total forward scattered light collected over a solid angle of $2 \pi$ steradians. An integrating sphere placed on the eyelid could, in principle, be used to realize the correct collection geometry, but, practically, the signal-tonoise ratio would be low and it would be difficult to fabricate and apply such a device to make reliable eyelid transmittance measurements.

For the purpose of this investigation, it was assumed that the transmitted light through the eyelid would follow a Lambertian angular distribution over the wavelength range of interest (i.e., 450 to $650 \mathrm{~nm}$ ) due to macromolecules (e.g., keratinocytes, collagen, and fat) in the skin. ${ }^{12}$ If the LED light source also has a Lambertian angular distribution and the field of view of the fiber-optic detector encompasses the full size of the LED source as well as the full size of the luminous spot of diffuse light emanating from the eyelid, then, given the same angular distribution of light with and without the eyelid, the total diffuse transmittance is simply equal to the amount of light measured after passing through the eyelid divided by the amount of light measured without the eyelid. ${ }^{13}$

The LED source chosen for the wand device had a radially symmetric, nearly Lambertian angular distribution. The cylindrical fiber-optic used in the wand device has a manufacturer specified (Newport Corp. model 77403) numerical aperture of 0.22 for incident irradiation (acceptance angle of approximately $25 \mathrm{deg}$ ). At the $12.5-\mathrm{mm}$ distance between the LED source and the fiber-optic input to the detector, it would be possible to collect light from the entire area of a 
luminous spot $5 \mathrm{~mm}$ in diameter. Thus, if the luminous spot size transmitted through the eyelid is effectively less than 5 $\mathrm{mm}$ in diameter and if the Lambertian angular distribution assumptions about the source and the eyelid media are strictly true, then the ratio of the transmittance measurements using an integrating sphere and the wand device would be equal to unity.

The accuracy of this logical construction was formally evaluated by comparing the spectral transmittance measurements of three (white, brown, and red) thin (all 0.4-mm thick) plastic diffusers taken by the wand device to those measured using an orthodox method employing collimated light and an integrating sphere. ${ }^{13}$ The orthodox spectral transmittance values ranged from 0.25 to less than $10^{-4}$ for the thin plastic samples, which is larger than the range of measured eyelid transmittance values described below. Averaging transmittance values across all wavelengths for the three different thin plastic samples, the orthodox method yielded mean \pm standard deviation (s.d.) transmittance values $1.2 \pm 0.1$ times higher than those obtained from the wand device. Transmittance values using the orthodox method and using the wand device for thick $(3.2 \mathrm{~mm})$ plastic diffusers were also compared. The orthodox method yielded mean \pm s.d. transmittance values for the thick samples that were $1.65 \pm 0.2$ times higher than those obtained from the wand device, presumably because the transmitted luminous spot size through the thick samples were effectively larger than $5 \mathrm{~mm}$ in diameter. Since the thickness of adult eyelids is approximately $0.5 \mathrm{~mm},{ }^{14}$ which is close to the thickness of the thin plastic diffusers $(0.4 \mathrm{~mm})$, a correction factor of 1.2 (20\% higher) was applied to all of the eyelid transmittance measurements reported below.

\section{Procedures}

Twenty-seven subjects (14 males; age range for all subjects was 18 to 52 years), recruited to have a wide range of apparent skin lightness participated in the experiment. The study was approved by Rensselaer Polytechnic Institute's Institutional Review Board (IRB). Upon arrival at the laboratory, subjects were informed about the measurement procedures and consented to participate.

For the eyelid spectral transmittance measurements, the subject was comfortably seated in a reclining chair for a period between 5 to $10 \mathrm{~min}$ in order to record up to four independent eyelid transmittance measurements. A computer controlled the application of electric power to the LED and the timing of the measurements. A registered nurse administered the measurement sequence. The measurement sequence began with an initial dark measurement to correct the subsequent measurements for thermal bias of the CCD detector. Next, the LED was energized and allowed to stabilize for $3 \mathrm{~s}$ before making the source measurement lasting $0.025 \mathrm{~s}$. The subject was asked to look down and the upper eyelid was then pulled gently away from the cornea by several millimeters. The LED strip was slid under the eyelid. Great care was taken when sliding the LED strip against the back of the eyelid in order to not touch the subject's cornea. Care was also taken when placing the small LED light source at the same location for every measurement. Specifically, the nurse attempted to locate the LED where the light would be transmitted through the center of the superior tarsus. The placement for measurement was deemed primarily relevant for estimating eyelid spectral transmittance as it would affect light passing through the iris pupil for retinal stimulation. After the LED strip was in position behind the eyelid, a sample measurement was taken. Since the radiant power of the optical signal transmitted through the eyelid was lower than during the source measurement, a signal integration period of $1.5 \mathrm{~s}$ was used for sample measurements. The spectral transmittance, $T(\lambda)$, was corrected by the ratio of source to sample times according to Eq. (1),

$$
T(\lambda)=\frac{P_{\text {Sample }}(\lambda)}{P_{\text {Source }}(\lambda)}\left(\frac{t_{\text {Source }}}{t_{\text {Sample }}}\right) C,
$$

where $t_{\text {Source }}$ and $t_{\text {Sample }}$ are the source and sample signal integration times, respectively, $P_{\text {Source }}$ and $P_{\text {Sample }}$ are the measured optical signals, and $C$, the diffuse transmittance measurement correction factor, equals 1.2.

The measurement sequence required approximately $10 \mathrm{~s}$ to complete, during which time the LED strip was behind the eyelid for $3 \mathrm{~s}$ or less. This sequence was repeated at a pace determined by the subject and the nurse.

In addition to eyelid spectral transmittance measurements, spectral reflectance measurements of the external eyelid skin were also obtained from a subset of 13 subjects ( 8 males; age range for the subset of subjects was 19 to 45 years), also recruited to have a wide range of apparent skin lightness. Diffuse illumination from white LED sources (GVA Lighting, Inc. model RGD-300), having a spectral output similar to that used in the wand device, were used for the spectral reflectance measurements. Subjects positioned their chins in a rest affixed to the threshold of a white hollow box $(0.6 \times 0.6 \times 0.6 \mathrm{~m})$ illuminated by the white LEDs. A small aperture on the opposite side of the box served as a port for the spectroradiometric measurements (Photo Research, Inc. model PR 705) of the spectral radiance of the eyelid and of a reflectance standard (Labsphere, Inc. model SRT-99-050) while subsequently positioned in the same location of the subject's closed eyelid. The spectral reflectance of the subject's eyelid was determined by dividing the eyelid spectral radiance measurements by the reflectance standard spectral radiance measurements for the matched wavelength intervals.

This same subset of 13 subjects was asked by the nurse to rate their level of pain during the eyelid spectral transmittance measurements using a scale from 1 (no pain) to 10 (unbearable pain).

\section{Results}

\subsection{Measurements}

The measured eyelid spectral transmittance curves for all 27 subjects are shown in Fig. 3(a). The mean \pm s.d. optical density of the eyelid from 450 to $650 \mathrm{~nm}$ was $2.1 \pm 0.3$ with an optical density range among subjects of approximately 1.0 across the entire spectral range. Each curve is the mean of two to four individual transmittance measurements for each subject. Eyelid transmittance is high at long wavelengths, dropping at approximately $600 \mathrm{~nm}$ and then again at approximately 515 $\mathrm{nm}$ as wavelengths become shorter. Although there are large individual differences in absolute eyelid transmittance, each of the curves has the same basic shape on a logarithmic ordinate, indicating that they are quite similar in terms of spectral 

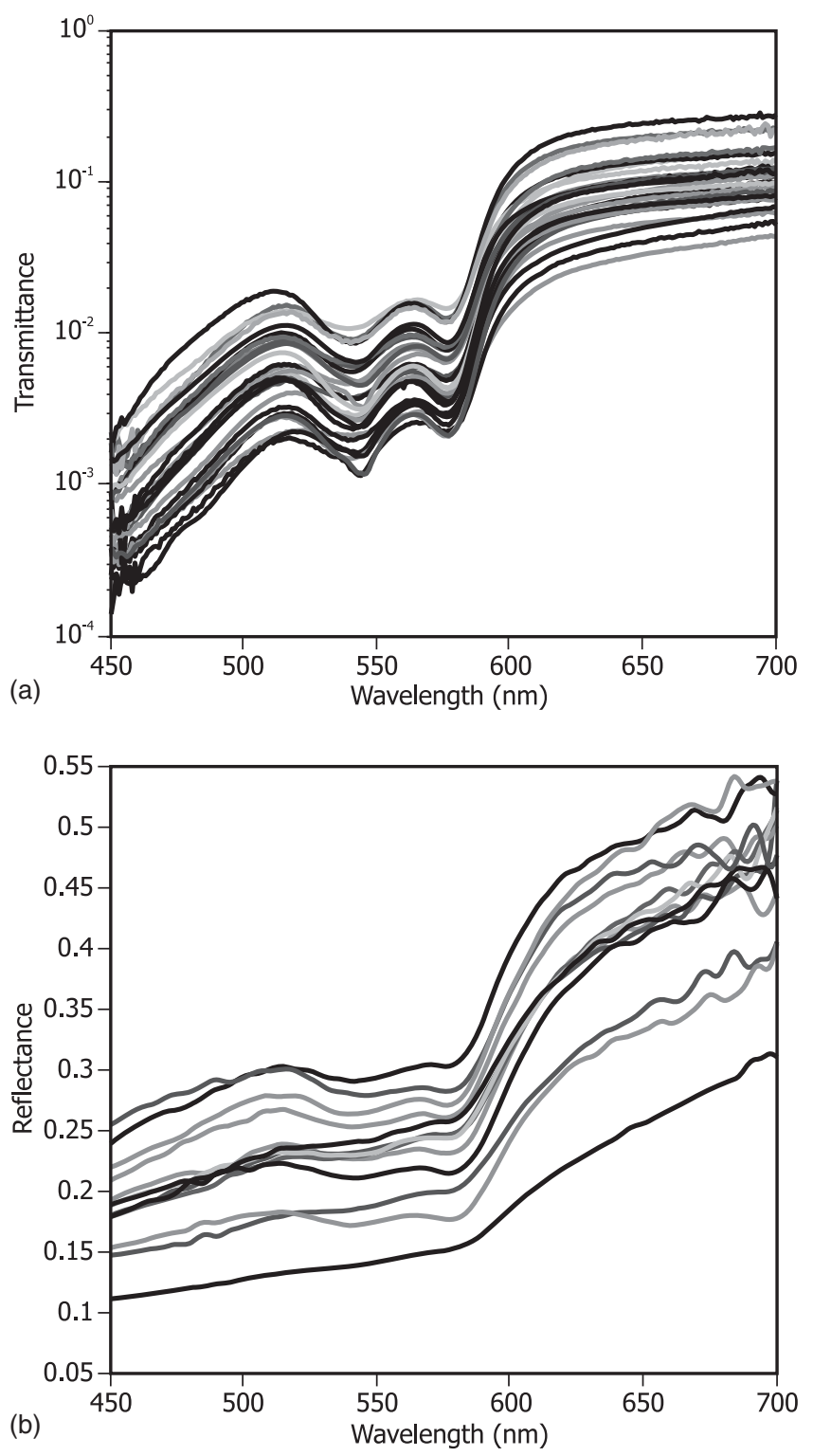

Fig. 3 (a) Eyelid spectral transmittance curves for 27 subjects. Each curve represents the mean of two to four independent spectral transmittance measurements obtained during a single session. (b) External eyelid skin spectral reflectance curves for the subset of 13 subjects. The white LED light sources in the hollow box used to illuminate the eyelids had low radiant power above $650 \mathrm{~nm}$ contributing to the less stable reflectance values at the longer wavelengths.

absorption. Thus, the individual differences must be largely due to wavelength-independent scatter.

Spectral reflectance measurements of the external eyelid skin for the subset of 13 subjects are shown in Fig. 3(b). A visual comparison between the curves in Figs. 3(a) and 3(b) shows that eyelid spectral transmittance cannot be accurately deduced from the eyelid spectral reflectance. In fact, the square of the sample correlation coefficients $\left(\mathrm{r}^{2}\right)$, calculated across subjects, between eyelid spectral transmittance and eyelid spectral reflectance at long wavelengths $(>650 \mathrm{~nm})$, are only approximately 0.1 . Even at short wavelengths $(<500 \mathrm{~nm})$, where the correlations are the highest, $\mathrm{r}^{2}$ values are never greater than 0.42 .

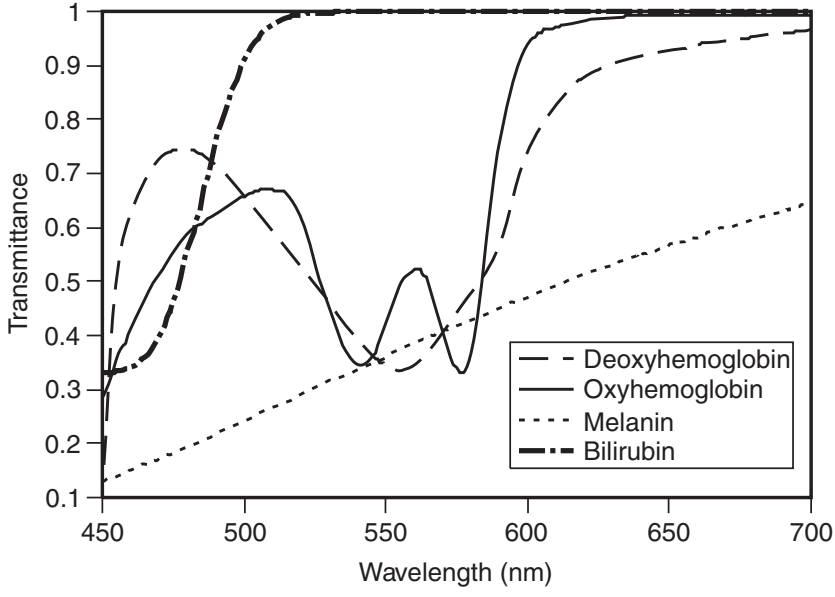

Fig. 4 Spectral transmittance of arbitrary unit amounts of hemoglobin, melanin, and bilirubin used for predicting the spectral transmittance of eyelid skin.

\subsection{Predicting Eyelid Transmittance}

The next goal was to develop a method for predicting eyelid spectral transmittance incorporating the known light absorbing constituents of skin. Spectral absorption data are readily available for the major light absorbing constituents of skin: blood, bilirubin, and the pigment melanin. ${ }^{11}$ The other constituents of the eyelid, keratinocytes, collagen, and fat exhibit very low light absorption characteristics, but reduce transmittance by scattering, thus exhibiting a large extinction coefficient.

The absorption coefficient of blood hemoglobin depends heavily on its oxygenated state and therefore must be broken down into two constituents: deoxy-hemoglobin and oxy-hemoglobin. These two constituents exhibit very strong absorption peaks at approximately $440 \mathrm{~nm}$ and at $420 \mathrm{~nm}$, respectively, and secondary absorption peaks at approximately $555 \mathrm{~nm}$ for deoxy-hemoglobin and 540 and $575 \mathrm{~nm}$ for oxy-hemoglobin. Tabulated values of absorption coefficients for deoxy-hemoglobin and for oxy-hemoglobin were obtained online from the Oregon Medical Laser Center website. ${ }^{15}$ In addition to deoxy-hemoglobin and oxy-hemoglobin, bilirubin, with a peak absorption band near $450 \mathrm{~nm}$, can also affect transmission through the eyelid. Figure 4 shows the absorption data for hemoglobin, melanin, and bilirubin converted to transmittance values from 450 to $700 \mathrm{~nm}$ in arbitrary unit amounts of substance. The concentrations of oxy- and deoxy-hemoglobin were set equal so that the modeled proportions could be easily compared with empirical proportions for skin from the literature.

Absorption of light by melanin is highly variable because there are many different types of melanin. ${ }^{10} \mathrm{In}$ fact, melanin is a broad class of different pigment compounds and the chemical makeup of melanin varies with genetic differences, different locations on the body, and for different amounts of previous ultraviolet (UV) exposure. The melanin curve shown in Fig. 4 represents the central tendency in skin melanin spectral transmittance and can be described by the following formula for the wavelength-dependent absorption coefficient, $\mu_{a}{ }^{16}$

$$
\mu_{a}=1.70 \times 10^{12} \lambda^{-3.48} \text { (in units of centimeters }{ }^{-1} \text { ). }
$$

$\lambda$ is wavelength in nanometers. 
To a first approximation, the nonpigmented constituents of the eyelid, particularly the dense connective tissue of the superior tarsus, should scatter light equally across the visible spectrum. Albino skin, for example, lacks melanin pigmentation and appears achromatic for light reflected off the bloodless skin surface. Therefore, for the purposes of predicting eyelid spectral transmittance, a single parameter based upon the scattering of visible optical radiation by eyelid, independent of wavelength, is simple and probably functionally representative of light scatter by macromolecules in skin and the superior tarsus, keratinocytes, collagen, and fat. ${ }^{12}$

The following equation for eyelid spectral transmittance incorporates the four key absorbing elements just described, deoxy-hemoglobin $(A)$ and oxy-hemoglobin $(B)$, melanin $(C)$, bilirubin $(D)$, as well as the nonabsorbing, scattering macromolecules $(e)$.

$$
T_{\lambda}=A_{\lambda}^{a} B_{\lambda}^{b} C_{\lambda}^{c} D_{\lambda}^{d} e
$$

$T_{\lambda}$, the eyelid transmittance, is a function of wavelength; $A_{\lambda}, B_{\lambda}$, $C_{\lambda}$, and $D_{\lambda}$ are the wavelength-dependent transmittance values for an arbitrary unit amount of substance, and the exponents $a$, $b, c$, and $d$ are the subject-specific variables to be determined, reflecting the amount of absorbing substance present in a person's eyelid. The factor $e$ is the wavelength-independent scattering variable.

Equation (3) was then transformed to eliminate the exponential dependency on the variables by taking the logarithm of both sides.

$$
\begin{aligned}
\log \left(T_{\lambda}\right)= & a \log \left(A_{\lambda}\right)+b \log \left(B_{\lambda}\right)+c \log \left(C_{\lambda}\right) \\
& +d \log \left(D_{\lambda}\right)+\log (e) .
\end{aligned}
$$

A regression analysis was used to estimate the values of the variables $a, b, c, d$, and $\log (e)$ for each of the subject-specific spectral transmittance curves in Fig. 3(a). To solve the equation, the transmittance values for each wavelength interval were substituted into Eq. (4). This method provided an over-determined system of 251 equations ( 450 to $650 \mathrm{~nm}$ every $1 \mathrm{~nm}$ ) for which optimal values of the variables $a, b, c, d$, and $\log (e)$ were found that minimized the squared error in the regression equation. Table 1 shows the results of the regression analysis for every constituent except bilirubin for every subject. Including or excluding bilirubin from the analysis had practically no effect on accurately predicting the spectral transmittance curves measured from any of the 27 subjects. It could be that the concentrations of bilirubin in the eyelid skins of these subjects were very low or that bilirubin absorption was masked by hemoglobin absorption, which is also quite high at wavelengths shorter than $500 \mathrm{~nm}$ (Fig. 4). Since including the spectral transmittance of bilirubin did not improve the curve fits, the final regressions did not include bilirubin. It is interesting to note in Table 1 that the ratio of oxy-hemoglobin to total hemoglobin for each subject falls within the range of 0.49 to 0.73 with a mean ratio of 0.65 , which is similar to the range of published values ( 0.37 to 0.72 ) for blood oxygen saturation levels for human skin tissue. ${ }^{17}$

Overall, except near the extreme ends of the utilized wavelength range, the matches between the measured and the predicted values were within $0.1 \log$ units $(25 \%)$ for each subject. Mismatches at short wavelengths can be attributed to stray light

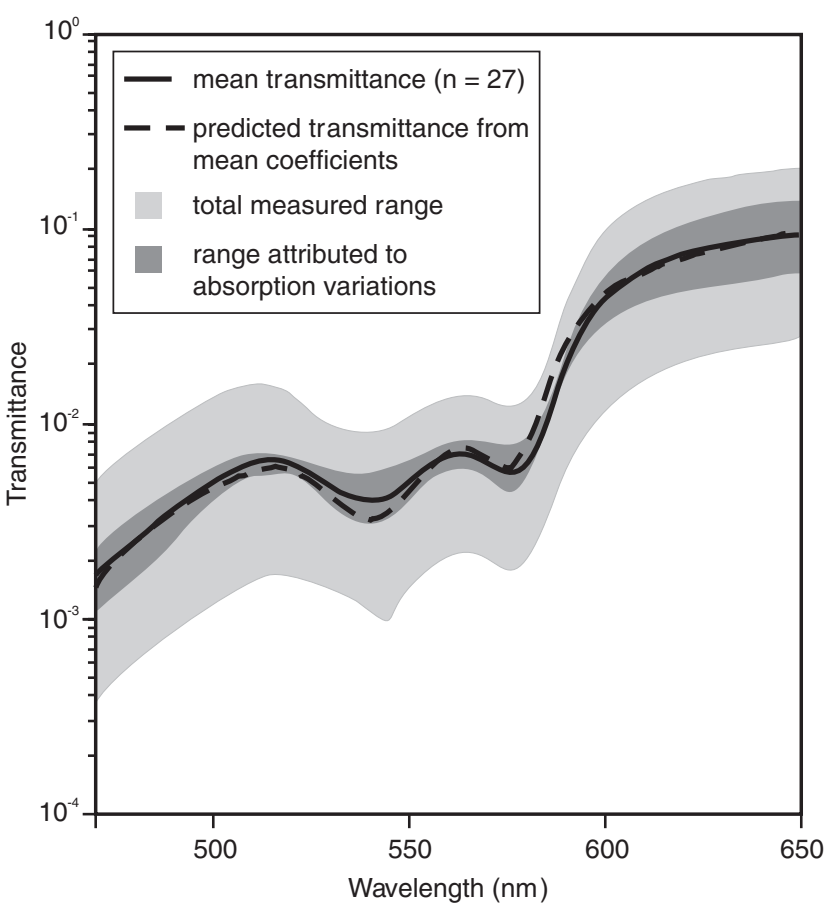

Fig. 5 The measured mean spectral transmittance function (solid line) calculated as the mean transmittance value at every wavelength increment for all 27 subjects and the predicted mean transmission function (dashed line) calculated from the mean of the wavelength-dependent fitting coefficients $(a, b, c)$ and scaled for a least-squares best fit with the measured mean transmittance curve to account for the wavelengthindependent scatter. The entire range of spectral transmittance values measured in the present study is illustrated by the light gray band, and the range attributable to absorption variations among subjects, i.e., not including wavelength-independent scatter, is shown by the darker gray band. The mean optical density of the eyelid from 450 to $650 \mathrm{~nm}$ was 2.1 with an optical density range among subjects of approximately 1.0 across this wavelength range.

and a low signal-to-noise ratio, resulting in erroneously high transmittance values as the signal-to-noise ratio approaches 1 . Mismatches at long wavelengths appear to be more systematic in that the measured spectral transmittance had less of an upward slope than the predicted spectral transmittance. Since light absorption by blood is negligible for these long wavelengths and wavelength-dependent scatter would cause deviations in the other direction (i.e., scatter generally decreases for increasing wavelength), this error may be attributable to any of the following: differing absorption values for melanin, other unidentified compounds in eyelid, or spectrally dependent anisotropic scattering.

Figure 5 shows the measured mean (solid line) and the predicted mean (dashed line) eyelid spectral transmission functions. The measured mean function represents the mean eyelid transmission values measured at each wavelength interval for each of the 27 subjects in the experiment. The predicted mean function reflects the means of the three major spectrally dependent coefficient values in Eq. (4) (one each for deoxy-hemoglobin, oxy-hemoglobin, and melanin as shown in Table 1) derived for each subject from their respective measured spectral transmission values and then scaled by a least-squares fit coefficient representing the spectrally uniform scattering. 
Table 1 Fitting coefficients $(a, b, c)$ and offset $(e)$ for predicting eyelid spectral transmittance.

\begin{tabular}{|c|c|c|c|c|}
\hline Subject no. & a (deoxy-hemoglobin) & b (oxy-hemoglobin) & $c$ (melanin) & $\log (e)$ (scatter) \\
\hline 1 & 0.677 & 1.731 & 2.830 & -0.775 \\
\hline 2 & 0.875 & 1.648 & 3.002 & -0.420 \\
\hline 3 & 0.788 & 0.742 & 2.855 & -1.936 \\
\hline 4 & 0.835 & 1.266 & 2.264 & -1.080 \\
\hline 5 & 0.849 & 1.223 & 2.987 & -1.635 \\
\hline 6 & 0.676 & 1.167 & 2.354 & -1.082 \\
\hline 7 & 0.695 & 1.652 & 3.086 & -1.054 \\
\hline 8 & 0.591 & 1.411 & 2.540 & -0.274 \\
\hline 9 & 0.969 & 1.440 & 3.079 & -0.877 \\
\hline 10 & 0.547 & 1.092 & 2.648 & -1.178 \\
\hline 11 & 0.759 & 1.642 & 2.796 & -1.431 \\
\hline 12 & 0.624 & 1.510 & 2.459 & -0.700 \\
\hline 13 & 0.694 & 1.088 & 2.755 & -1.199 \\
\hline 14 & 0.540 & 0.936 & 2.146 & -1.036 \\
\hline 15 & 0.984 & 1.494 & 2.106 & -0.308 \\
\hline 16 & 0.821 & 1.430 & 2.659 & -0.867 \\
\hline 17 & 0.910 & 1.216 & 2.513 & -0.629 \\
\hline 18 & 0.738 & 1.278 & 2.544 & -0.324 \\
\hline 19 & 0.600 & 1.267 & 3.381 & -1.018 \\
\hline 20 & 0.481 & 1.327 & 2.072 & -1.074 \\
\hline 21 & 0.671 & 1.160 & 2.858 & -0.743 \\
\hline 22 & 0.796 & 1.277 & 2.328 & -0.890 \\
\hline 23 & 0.969 & 1.666 & 2.881 & -1.005 \\
\hline 24 & 0.536 & 1.368 & 2.932 & -0.971 \\
\hline 25 & 0.812 & 1.592 & 2.097 & -1.183 \\
\hline 26 & 0.673 & 1.552 & 2.536 & -1.145 \\
\hline 27 & 0.853 & 1.750 & 2.661 & -0.716 \\
\hline Mean & 0.739 & 1.368 & 2.643 & -0.825 \\
\hline Std. deviation & 0.142 & 0.251 & 0.343 & 0.397 \\
\hline Std. deviation/mean (\%) & 19 & 18 & 13 & 48 \\
\hline
\end{tabular}


The light gray band in Fig. 5 reflects the entire range of eyelid spectral transmittance values measured in the present study. To gain insight on how much of the transmittance variation among subjects was due to wavelength-independent scatter and how much was due to the absorbing constituents, the predicted mean function was fitted to each subject's measured data using a single scaling coefficient representing wavelength-independent scatter. The spread of values about the measured mean spectral transmittance curve, shown as the dark gray band in Fig. 5, represents the variation among subjects attributable to differences in wavelength-dependent absorption. As can be readily appreciated from Fig. 5, when the variation due to nonspectrally selective phenomena (scattering) is removed, eyelid spectral transmittance is quite similar among the sample of subjects employed in the present experiment, implying that most of the absolute variation in eyelid spectral transmittance is due to nonabsorbing macromolecules in the eyelid. This point is reinforced by the last row of values in Table 1 . The nonabsorbing scatter coefficient has the largest relative variance and the pigment melanin has the smallest. Thus, in terms of eyelid transmission, eyelid thickness, and/or the density of macromolecules in the eyelid account for the largest variation among subjects.

Subjectively, the subset of 13 subjects all reported that the level of pain experienced during the eyelid transmittance measurements was 1 , which means no one experienced pain during data collection.

\section{Discussion}

Reported here are data acquired using a technique that was developed to provide practical and accurate measurements of eyelid transmittance across the visible portion of the spectrum. Directly placing a very small, calibrated source behind the eyelid, at the center of the superior tarsus with a fixed detector in front, provided greater measurement sensitivity than could be obtained from previously reported methods. Importantly, too, the measurements could be obtained quickly and with no physical discomfort to the subjects.

For long-wavelength visible radiation transmitted through the eyelid where the measurement signal-to-noise ratio is relatively high, the results of previous studies agree well with the present results. For example, a mean estimate of $9 \%$ transmittance at $630 \mathrm{~nm}$ was obtained in the present study. Using a psychophysical technique and a red LED with an emission band between 615 to $635 \mathrm{~nm}$, Ando and Kripke ${ }^{5}$ estimated a mean transmission of $5.6 \%$. Objective methods employed by Robinson et al. ${ }^{4}$ and by Moseley et al. ${ }^{3}$ revealed $9 \%$ and $8.5 \%$ transmission, respectively, at $630 \mathrm{~nm}$. Given the wide range of absolute transmission values among individuals, these estimates are remarkably consistent. For shorter wavelengths, agreement between the methods greatly diminishes. Based upon their psychophysical method, Ando and Kripke report a mean transmission of $0.3 \%$ for both middle wavelengths (540 to $565 \mathrm{~nm}$ ) and short wavelengths (400 to $510 \mathrm{~nm}$ ). Using objective methods, Robinson et al. and Moseley et al. show mean transmittance values between 1 and $2 \%$ over most of this spectral range with an increase in eyelid transmittance as wavelength decreases to $400 \mathrm{~nm}$. At first glance, these data seem suspect from a low signal-to-noise artifact. ${ }^{9}$ The present objectively measured data agree better with the psychophysical data; mean transmittance at $550 \mathrm{~nm}$ was approximately $0.5 \%$ and $0.4 \%$ at $490 \mathrm{~nm}$. Importantly, the present study demonstrates decreasing transmittance as wavelength decreases below $515 \mathrm{~nm}$. Bolstered by the consistent results from the regression analyses (Fig. 5), the present measurement technique appears to provide greater accuracy in estimating the eyelid spectral transmittance than has previously been reported, particularly at short wavelengths where absorption by hemoglobin (and perhaps bilirubin) is quite high.

This study also shows that external skin pigmentation is not highly correlated with eyelid transmission. No more than $40 \%$ of the variance can be explained by absorption, and only at short wavelengths where light absorption is highest. Although melanin absorbance is primarily responsible for differences in external skin reflectance, the modeled values for its impact on eyelid transmittance did not vary much among subjects. Most of the variance in the modeled eyelid spectral transmittance among the subjects studied was attributed to macromolecule (i.e., not wavelength specific) scattering in the eyelid. This suggests that eyelid thickness and/or the densities of keratinocytes, collagen, and fat ${ }^{12}$ are primarily responsible for absolute eyelid transmission for most of the visible spectrum, although this was not formally tested.

The ability to more accurately estimate the absolute and spectral transmission of a person's eyelid may be important for applications where light might be delivered through closed eyes. For example, the high optical density (or low transmittance) at short wavelengths (below about $515 \mathrm{~nm}$ ) has particular relevance for quantifying how light filtered through the eyelids might stimulate the human circadian system which has peak sensitivity in this region of the spectrum. ${ }^{18}$ Exposure to light at night can suppress the hormone melatonin, which is produced at night and under conditions of darkness. Animal studies have shown that melatonin reduces the rate of tumor growth. For humans, although a direct relationship between light at night exposure and breast cancer initiation has not been established, it has been hypothesized that chronic melatonin suppression by light at night can be a health risk. Therefore, quantifying the impact of any light that passes through the closed eyelids would be important for discussions concerning the health impacts of light at night. Also, circadian sleep disorders, such as delayed sleep phase, might be effectively treated if light is delivered through closed eyelids close to the minimum core body temperature, when phase shifting effects of light is greatest. ${ }^{2,19}$ Estimates of light transmission for the visual system are also of interest. One recent study, for example, estimated visual spatial resolution and direction discrimination using red light from an $\operatorname{LED}\left(\lambda_{\max }=635 \mathrm{~nm}\right)$ applied through closed eyes. ${ }^{20}$

In general, from the predicted spectral transmittance function developed in the present study, it now should be possible to closely estimate eyelid transmittance at any wavelength, including short wavelengths, which have been difficult to measure accurately. More specifically, the effectiveness of a light source of known spectral power distribution incident on the closed eyelid can be determined by weighting it by the proposed eyelid spectral transmittance function to fairly accurately estimate the relative transmittance of the eyelid for individuals, regardless of differences in skin reflectance. To obtain an estimate of absolute transmittance through the eyelid, however, a measurement must be made of an individual's absolute transmittance at a specific wavelength in the visible band (preferably long wavelengths 
where the eyelid has high transmission). From that measurement the predicted eyelid spectral transmittance can be used to estimate the absolute transmittance at any other wavelength within the visible band because, to a first approximation, an individual's large molecule, the achromatic scattering coefficient, simply modulates the transmittance at every wavelength by the same factor.

\section{Acknowledgments}

The authors are grateful for the support of Michael Colbaugh of Philips Respironics, and Barbara Plitnick and Robert Hamner of the Lighting Research Center, Rensselaer Polytechnic Institute.

\section{References}

1. R. G. Foster and K. Wulff, "The rhythm of rest and excess," Nat. Rev. 6(5), 407-414 (2005).

2. R. J. Cole, J. S. Smith, Y. C. Alcalá, J. A. Elliott, and D. F. Kripke, "Bright-light mask treatment of delayed sleep phase syndrome," J. Biol. Rhythms. 17(1), 89-101 (2002).

3. M. J. Moseley, S. C. Bayliss, and A. R. Fielder, "Light transmission through the human eyelid: in vivo measurement," Ophthalmic Physiol. Opt. 8, 229-230 (1988).

4. J. Robinson, S. C. Bayliss, and A. R. Fielder, "Transmission of light across the adult and neonatal eyelid in vivo," Vision Res. 31(10), 1837-1840 (1991).

5. K. Ando and D. Kripke, "Light attenuation by the human eyelid," Biol. Psychiatry 39(1), 22-25 (1996).

6. A. N. Bashkatov, E. A. Genina, V. I. Kochubey, and V. V. Tuchin, "Optical properties of human skin, subcutaneous, and mucous tissues in the wavelength range from 400 to $2000 \mathrm{~nm}$," J. Phys. D: Appl. Phys. 38, 2543-2555 (2005).

7. I. V. Meglinski and S. J. Matcher, "Quantitative assessment of skin layers absorption and skin reflectance spectra simulation in the visible and near-infrared spectral regions," Physiol. Meas. 23, 741-753 (2002).
8. I. V. Meglinski and S. J. Matcher, "Computer simulation of the skin reflectance spectra," Comput. Methods Programs Biomed. 70, 179-186 (2003).

9. H. J. Kostkowski, Reliable Spectroradiometry. pp. 57-58, Spectroradiometery Consulting, La Plata (1997).

10. T. Igarashi, K. Nishino, and S. K. Nayar, "The appearance of human skin," Technical Report: CUCS-024-05, Columbia University, New York (2005).

11. R. R. Anderson and J. A. Parrish, "The optics of human skin," J. Invest. Dermatol. 77, 13-19 (1981).

12. J. A. McGrath , R. A. J. Eady, and F. M. Pope, "Anatomy and organization of human skin," Chapter 3, in Rook's Textbook of Dermatology, 7th ed., T. Burns, S. Breathnach, N. Cox, and C. Griffiths, Eds., Blackwell Publishing, Massachusetts (2004).

13. J. W. T. Walsh, Photometry. pp. 433-435, Constable and Company Ltd., London (1958).

14. Y. Lee and K. Hwang, "Skin thickness of Korean adults," Surg. Radiol. Anat. 24, 183-189 (2002).

15. S. A. Prahl, "Tabulated molar extinction coefficient for hemoglobin in water," Oregon Medical Laser Center, 1998, accessed June 2010 at http://omlc.ogi.edu/spectra/hemoglobin/summary.html.

16. S. L. Jacques, "Melanosome absorption coefficient," Oregon Medical Laser Center, 1998, accessed June 2010 at http://omlc.ogi.edu/spectra/ melanin/mua.html.

17. I. C. Roddie, J. T. Shepherd, and R. F. Whelan, "Evidence from venous oxygen saturation measurements that the increase in forearm blood flow during body heating is confined to the skin," J. Physiol. 134(2), 444-450 (1956).

18. M. S. Rea, M. G. Figueiro, J. D. Bullough, and A. Bierman, "A model of phototransduction by the human circadian system," Brain Res. Rev. 50, 213-228 (2005)

19. J. M. Zeitzer, D. J. Dijk, R. Kronauer, E. Brown, and C. Czeisler, "Sensitivity of the human circadian pacemaker to nocturnal light: melatonin phase resetting and suppression," J. Physiol. 526(3), 695-702 (2000).

20. A. Pelah, S. Liu, M. Gilbert, H. Hock, and P. Jepson, "Reflections on the eyelid: experiencing structured light through closed eyes," in Proc. of Experiencing Light 2009, pp. 155-161, Eindhoven University of Technology, Eindhoven (2009). 